\title{
BMJ Open Association between postsurgical pain and heart rate variability: protocol for a scoping review
}

\author{
Vincent So, ${ }^{1}$ Gregory Klar, ${ }^{1}$ Jordan Leitch, ${ }^{1}$ Michael McGillion, ${ }^{2}$ P J Devereaux, ${ }^{3}$ \\ Ramiro Arellano, ${ }^{1}$ Joel Parlow, ${ }^{1,4}$ Ian Gilron (1) 1,5,6
}

To cite: So V, Klar G, Leitch J, et al. Association between postsurgical pain and heart rate variability: protocol for a scoping review. BMJ Open 2021;11:e044949. doi:10.1136/ bmjopen-2020-044949

- Prepublication history and additional supplemental material for this paper are available online. To view these files, please visit the journal online (http://dx.doi.org/10.1136/ bmjopen-2020-044949)

Received 17 September 2020 Revised 29 March 2021 Accepted 30 March 2021

Check for updates

(C) Author(s) (or their employer(s)) 2021. Re-use permitted under CC BY-NC. No commercial re-use. See rights and permissions. Published by BMJ.

For numbered affiliations see end of article.

Correspondence to

lan Gilron; gilroni@queensu.ca

\section{ABSTRACT}

Introduction Surgical interventions can elicit neuroendocrine responses and sympathovagal imbalance, ultimately affecting cardiac autonomic function. Cardiac complications account for $30 \%$ of postoperative complications and are the leading cause of morbidity and mortality following non-cardiac surgery. One cardiovascular parameter, heart rate variability (HRV), has been found to be predictive of postoperative morbidity and mortality. HRV is defined as variation in time intervals between heartbeats and is affected by cardiac autonomic balance. Furthermore, altered HRV has been shown to predict cardiovascular events in non-surgical settings. In multiple studies, experimentally induced pain in healthy humans leads to reduced HRV suggesting a causal relationship. In a different studies, chronic pain has been associated with altered HRV, however, in the setting of clinical pain conditions, it remains unclear how much HRV impairment is due to pain itself versus autonomic changes related to analgesia. We aim to review the available evidence describing the association between postsurgical pain and HRV alterations in the early postoperative period. Methods and analysis We will conduct a scoping review of relevant studies using detailed searches of MEDLINE and EMBASE, in accordance with the Preferred Reporting Items for Systematic Reviews and Meta-Analysis. Included studies will involve participants undergoing non-cardiac surgery and investigate outcomes of (1) measures of pain intensity; (2) measures of HRV and (3) statistical assessment of association between \#1 and \#2. As secondary review outcomes included studies will also be examined for other cardiovascular events and for their attempts to control for analgesic treatment and presurgical HRV differences among treatment groups in the analysis. This work aims to synthesise available evidence to inform future research questions related to postsurgical pain and cardiac complications.

Ethics and dissemination Ethics review and approval is not required for this review. The results will be submitted for publication in peer-reviewed journals.

\section{BACKGROUND}

Postoperative cardiac complications in noncardiac surgery

Annually, over $4 \%$ of the world's population ( 200 million adults) undergo non-cardiac surgery. ${ }^{1}$ Unfortunately, following non-cardiac

\section{Strengths and limitations of this study}

There are currently no reviews synthesising evidence of the relationship between postoperative pain and heart rate variability, which is likely relevant to the risk of postoperative cardiovascular complications.

- Our study includes a comprehensive and systematic literature search and detailed assessment of bias in accordance with the Preferred Reporting Items for Systematic Reviews and Meta-Analysis statements and the predefined methodology based on the Cochrane Handbook for Systematic Reviews of Interventions.

- Diverse studies included in this review may be heterogeneous with respect to various factors.

surgery, $7 \%-11 \%$ of patients experience postoperative complications, most of which $(\sim 30 \%-40 \%)$ are cardiac-related. ${ }^{2-4}$ Additionally, postoperative complications result in a mortality rate of $0.8 \%-1.5 \%,{ }^{56}$ and are the third leading cause of death in the US. ${ }^{7}$

Although postoperative cardiac risk varies substantially based on surgical factors such as invasiveness, type of surgery, duration of procedure and blood loss, it is important to consider the stress response that occurs following surgery. ${ }^{6}{ }^{8}$ For example, surgical interventions produce tissue injury that elicits neuroendocrine responses and sympathovagal imbalance. ${ }^{68}$ Other surgical stresses come from anesthesia-related physiologic perturbations, acute anaemia, hypercoagulability, blood pressure changes, fluid shifts and hypothermia. ${ }^{7}$ These stressors can increase myocardial oxygen demand and lead to haemodynamic derangements, ultimately resulting in various cardiac complications especially in patients with pre-existing cardiovascular risk factors. ${ }^{6}$ Some postoperative cardiac complications include perioperative myocardial infarction (PMI), cardiac arrest, congestive heart failure ${ }^{7}$ and myocardial injury after non-cardiac surgery (MINS), with 
MINS being the most common postoperative cardiovascular complication. ${ }^{41011}$

\section{Predictors of adverse postsurgical cardiovascular events}

Practice guidelines currently suggest routine postoperative assessment of cardiac troponin levels for patients with cardiovascular risk factors, mainly to detect PMI and MINS. The rationale for these guidelines is that elevated troponin concentration is a sensitive and specific biomarker for myocardial injury, and have also been shown to predict 30-day and 1-year mortality in patients undergoing non-cardiac surgery. ${ }^{6}$ 12-14 Specifically, the diagnosis of MI requires elevated troponin levels (above 99th percentile) accompanied by characteristic chest pain, new ST segment changes or left bundle branch block, ventricular wall motion abnormalities or intracoronary thrombus on angiography. ${ }^{15}$ In contrast to non-operative patients, postoperative patients receiving analgesia do not commonly experience chest pain typical of MI and do not always show pathognomonic ECG changes. ${ }^{2}$ In fact, in one study by Puelacher et al, PMI was only accompanied by typical chest pain in $6 \%$ of patients, and ischaemic symptoms in $18 \%$ of patients. ${ }^{16}$

Since many patients sustaining myocardial injury in the postoperative period do not meet the diagnostic criteria for MI, a new diagnosis has been established for patients with elevated troponin, irrespective of the presence of ischaemic symptoms or electrocardiographic findings, known as MINS. ${ }^{4}$ MINS is believed to be due to an ischaemic aetiology, and requires exclusion of non-ischaemic aetiology such as rapid atrial fibrillation, sepsis and pulmonary embolism as the underlying cause of abnormalities. In one large cohort study, elevated troponin levels judged due to an ischaemic aetiology (meeting MINS criteria) was an independent predictor of 30-day mortality. ${ }^{4}$ Importantly, an international, randomised controlled trial conducted in 2018 demonstrated that treatment with anticoagulant therapy (dabigatran $110 \mathrm{mg}$ twice daily) can lower the risk of major cardiovascular complications for patients with MINS, suggesting that the suboptimal prognosis of MINS is modifiable. ${ }^{17}$

More recently, a meta-analysis conducted in 2019 by Zhang et al suggested that various cardiac biomarkers are predictive of postoperative major adverse cardiovascular events (MACE) in patients undergoing non-cardiac surgery. ${ }^{18}$ The definition of MACE included a variety of cardiovascular conditions of various ischaemic and nonischaemic etiologies. ${ }^{18}$ In this study, various biomarkers such as elevated levels of brain natriuretic peptide, high sensitivity C-reactive protein and high-sensitivity cardiac troponin $\mathrm{T}$ were shown to lead to up to 4.5 -fold increase, 4-fold increase and 6-fold increase in the risk of MACE, respectively. ${ }^{18}$ These findings suggest that these various biomarkers can predict cardiovascular outcomes that are not necessarily due to ischaemic etiologies (as presumed in MINS), such as all-cause mortality, heart failure and arrhythmias. Taken together, there are various biomarkers of postsurgical cardiovascular events, but other predictive factors should be explored to further guide cardiac prevention efforts and provide additional prognostic value in the postsurgical setting for adverse cardiovascular events.

\section{Heart rate variability as a predictor of adverse cardiovascular events}

Healthy individuals exhibit a rhythmic variation in time intervals from one R wave to the next on ECG. Heart rate variability (HRV) is defined as the pattern of variation in the R-R time interval between heartbeats. HRV can be subdivided into time-domain indices and frequencydomain values, both of which are linear phenomena. ${ }^{19}$ The time domain indices quantify the amount of HRV observed during monitoring periods. ${ }^{19}$ In contrast, frequency-domain values represent the absolute or relative amount of signal energy within component bands, and can be further subdivided into high frequency (HF; $0.20-0.40 \mathrm{~Hz}$ ) and low frequency (LF; $0.04-0.15 \mathrm{~Hz}$ ) components following spectral analysis. ${ }^{20}$ Interestingly, variability in $\mathrm{HF}$ components reflects changes in the parasympathetic nervous system (PNS). On the other hand, LF variability may indicate changes in both the PNS and sympathetic nervous system (SNS), ${ }^{20}$ although the utility of this measurement is heavily debated and highly dependent on data collection procedures. ${ }^{21}$ Taken together, HRV is an important measure of PNS (and possibly the balance between PNS and SNS), and may serve as an indicator of autonomic balance. ${ }^{20}$

Of relevance to this review, various comorbid conditions-as well as medications used during the perioperative period-have been associated with altered HRV, including general anaesthetics, ${ }^{22}{ }^{23}$ anticholinergic agents, ${ }^{24}$ antihypertensive agents, ${ }^{25}$ antihistamines ${ }^{26}$ and beta-blockers. ${ }^{27}$ Recently, HRV has been proposed as a tool to measure the physiological stress response during general anaesthesia, as well as in the postoperative period. ${ }^{20}$ Similar to troponin measurements, low HRV has been shown to independently predict postoperative morbidity and long-term mortality. ${ }^{312} 2829$ Additionally, depressed HRV before induction of anaesthesia was shown to be predictive of 30-day mortality in the postsurgical setting. ${ }^{12} 28$ These data suggest that HRV may be a useful tool to detect autonomic instability in the preoperative and early postoperative setting and may be useful for identifying patients who are at high risk for poor postoperative outcomes due to low autonomic physiology reserves.

\section{Pain and anesthetic agents alter heart rate variability}

Given that the autonomic nervous system is significantly affected by the experience of pain, ${ }^{30} 31$ it is likely that autonomic parameters such as HRV are altered in the setting of pain. In support of this notion, HRV changes have been reported in a variety of patients with chronic pain conditions, ${ }^{32}$ such as breakthrough pain in cancer, ${ }^{33}$ complex regional pain syndrome,${ }^{34}$ fibromyalgia ${ }^{35}$ and chronic neck pain. ${ }^{36}$ 
In contrast, there are fewer studies looking at the relationship between HRV and acute pain or nociception in healthy adults. ${ }^{37}$ Nevertheless, studies have suggested that high-frequency HRV is strongly correlated with pain intensity in both adults and children. ${ }^{38}{ }^{39}$ In addition, healthy patients with selfreported symptoms of pain may have lower parasympathetic activity and altered HRV. ${ }^{40}$ In another study by Treister $e t$ al the authors demonstrated that decreased HRV (HF component) could differentiate between painful stimuli and non-painful stimuli, although HRV alone could not discriminate between differences in pain intensity (low, medium or high pain categories) ${ }^{41}$ However, in this same study, the linear combination of the multiple autonomic parameters including HRV, heart rate, skin conductance levels and fluctuations and photoplethysmographic pulse wave amplitude, differentiated both the presence of pain and could discriminate between the different pain categories. ${ }^{41}$ Moreover, studies have suggested that greater HRV (LF measurements) are associated with higher thresholds for pain, ${ }^{42}$ although the utility of LF HRV measurements are highly disputed and should be interpreted with caution. ${ }^{21}$

In addition to acute and chronic pain conditions, changes in HRV have also been observed following the administration of pharmacologic agents for acute pain management and anaesthesia. For example, the administration of spinal anaesthesia (isobaric bupivacaine) has been shown to significantly decrease the $\mathrm{LF} / \mathrm{HF}$ ratio of $\mathrm{HRV}^{43}$ This may be due to a shift in the balance towards the parasympathetic system, related to the sympathetic block caused by spinal anaesthesia. Interestingly, in the same study, the change in LF/ HF was attenuated by coadministering intrathecal fentanyl, providing further evidence that opioid medications (eg, fentanyl) commonly used for pain management can have direct effects on HRV. ${ }^{43}$ Other studies support the notion that induction of anaesthesia can alter HRV, with decreases in non-linear HRV indices (approximate entropy, peak approximate entropy and point correlation dimension) following fentanyl-based induction of anaesthesia. ${ }^{44}$ Likewise, there is evidence that various anaesthetic agents such as general anaesthesia, ${ }^{45}$ propofol, ${ }^{23} 46$ isoflurane ${ }^{47}$ and sevoflurane ${ }^{22}$ can also alter HRV following administration. Taken together, these studies suggest that pain is associated with changes in the autonomic nervous system, and autonomic measures such as HRV can be altered in the acute and chronic pain setting, as well as during the use of opioids.

\section{Rationale for studying the association between heart rate variability and postsurgical pain management}

Given emerging evidence that pain as well as pain medications such as opioids have pronounced respiratory, cardiovascular and autonomic effects, ${ }^{48}$ and pain has been shown to influence cardiac autonomic nervous system indices, it is critical to review the current evidence so as to guide future research efforts to better understand the relationship between altered HRV and postsurgical pain. Therefore, the evidence surrounding a possible association between postsurgical pain and HRV, which could ultimately influence the risk for postoperative cardiovascular complications, is highly relevant.

\section{Objectives and research question}

The aim of this scoping review is to synthesise and review studies describing the association between postsurgical pain and HRV in patients undergoing non-cardiac surgery. A secondary aim is to investigate cardiovascular outcomes in relation to HRV measurements and postsurgical pain, as well as to investigate a study's attempts to control for analgesic treatment, and presurgical differences in HRV in the data analysis.

\section{METHODS}

This protocol was written in accordance with the Preferred Reporting Items for Systematic Reviews and Meta-Analysis (PRISMA-P). ${ }^{50}$

\section{Study selection}

Types of studies

We will include all study types with primary data available (no review articles) published in a peer-reviewed journal. To minimise the risk of publication bias (small study bias), ${ }^{51}$ any studies with less than 10 participants will be excluded.

\section{Patient population}

We will include studies involving adults aged 18 years and over who are undergoing non-cardiac surgery, regardless of the presence or absence of cardiovascular risk factors. Studies must include patients who have had HRV measured and who have undergone assessment for postsurgical pain (ie, using a validated measure of pain intensity or change in pain intensity (pain relief)) within the postoperative period (up to 30 days after surgery).

\section{Inclusion criteria}

1. Studies of any design that include measures of pain intensity or pain relief within the first 30 days after noncardiac surgery.

2. Pain intensity or pain relief quantified using a validated measurement instrument (eg, 0-10 numerical rating scale or $0-100 \mathrm{~mm}$ visual analogue scale for pain intensity; category scale for pain relief).

3. HRV measurements such as frequency bands, ratios of frequency bands, time indices of HRV and total power. Frequency bands include LF power $(0.04-0.015 \mathrm{~Hz})$, HF power $(0.15-0.45 \mathrm{~Hz})$ or ratios of $\mathrm{LF} / \mathrm{HF}$ or $\mathrm{HF} /$ LF. Time domain indices of HRV include SD of NN intervals, SD of the averages of NN intervals, square root of the mean of the sum of the squares of differences between adjacent NN intervals and SD of differences between adjacent $\mathrm{NN}$ intervals. 


\section{Exclusion criteria}

1. Animal studies (no human data).

2. Review papers (no primary data).

3. Cardiac surgery.

4. Studies not written in the English language.

\section{Identification of studies and search strategy}

We will conduct a detailed search on MEDLINE and EMBASE. Detailed searches will be conducted from the inception of the database until the date the searches are run (see online supplemental appendix 1). The search will include terms related to HRV, postsurgical pain, noncardiac surgery and relevant cardiovascular outcomes (eg, myocardial infarction, pulmonary embolism). The bibliography of identified articles will be cross-referenced to check for additional studies to include in the review. The search strategy will be developed in consultation with a librarian specialising in literature searches.

\section{Types of outcome measures}

\section{Primary outcomes}

1. Measures of pain intensity and/or changes in pain intensity (pain relief).

2. HRV within the first 30 days after non-cardiac surgery in humans.

3. Change from preoperative baseline HRV within the first 30 days after non-cardiac surgery in humans.

4. Statistical assessment of the association between (1) and (2) or between (1) and (3).

\section{Secondary outcomes}

1. Cardiovascular events (eg, myocardial infarction, stroke and pulmonary embolism).

2. Other autonomic parameters (eg, skin conductance level and fluctuations, photoplethysmographic pulse wave amplitude and catecholamine levels).

3. Use of analgesics and differences in analgesia between study groups.

\section{Data collection and extraction}

Two authors will independently evaluate studies for eligibility. Screening for eligibility of studies will be performed on titles and abstracts, followed by full-text screening for citations considered potentially eligible by either screener. All citations identified in the screening process as potentially eligible will undergo full text evaluation to determine eligibility by two independent reviewers. Any disagreements between the two reviewers will be resolved through discussion and consensus, and a third reviewer will be consulted if required. Following full-text review, data from eligible studies will be recorded using standardised extraction forms using the Covidence web source (www.COVIDENCE.org). The standardised forms will capture information about types of postsurgical pain, details of postsurgical pain management, pain intensity, cardiovascular risk factors, measures of HRV and participant characteristics. As an optional secondary outcome for the review, postoperative cardiovascular outcomes will be recorded if it is included in eligible studies.

\section{Risk of bias}

Risk of bias for each eligible study will be independent assessed by two reviewers using the criteria outlined in the Cochrane Handbook for Systematic Review of Interventions. ${ }^{52}$ For any study that includes multiple painrelated measures or interventions (eg, pain intensity or change in pain intensity), each measure will be assessed independently for risk of bias. Disagreements between the two reviewers will be resolved through discussion and consensus, and a third reviewer will be consulted if needed. Each category of bias will be assigned an unclear, low or high risk of bias and summarised in a risk of bias chart.

In each study, we will assess for the following risk of biases: (a) selection bias due to incomplete data collection, (b) incomplete outcome data due to lost to follow-up for risk for attrition bias, (c) selective reporting for detection bias, (d) number of participants for possible biases (eg, publication bias) that are confounded by small sample size, (e) information bias (including recall and observer biases) to address how data are obtained from study groups, which will be especially important for studies with non-randomised interventions and (f) confounding bias due to differences in comorbidities, demographic and surgical characteristics, baseline HRV differences, differences in analgesic use and other patient factors between study groups.

\section{Analysis plan}

A descriptive approach will be used to report primary and secondary outcomes due to the variation which will likely exist across identified studies. For studies that are similar with respect to study design, participant population, measures used and analysis methods for the association between pain and HRV, meta-analysis will be performed in consultation with a biostatistician.

\section{Patient and public involvement}

No patients involved.

\section{DISCUSSION}

Cardiovascular complications are a common cause of morbidity and mortality in the postoperative setting. ${ }^{2-4}$ Among several cardiovascular factors, HRV has been shown to be an independent predictor of postoperative morbidity and long-term mortality following non-cardiac surgery. $^{312} 2829$ In general, abnormal HRV reflects autonomic imbalance and has been associated with anaesthetic use, ${ }^{22} 2347$ chronic pain conditions ${ }^{33-35}$ and acute experimental pain in healthy patients. ${ }^{37} 40-42$ Despite the welldocumented relationship between postsurgical outcomes and HRV, and the presence of HRV in various pain conditions, there has not been a review of available evidence describing the association between postsurgical pain and HRV. This scoping review aims to synthesise information surrounding the relationship between postsurgical pain and HRV, which may have important implications for 
adverse cardiovascular outcomes following non-cardiac surgery.

In summary, this scoping review will explore the association between HRV and postsurgical pain and pain management. Depending on the identified studies and the data available, associations between HRV and postsurgical cardiovascular outcomes may also be assessed, with the overall aim to inform future research questions to better understand cardiovascular outcomes following non-cardiac surgery.

\section{Limitations and challenges}

The strengths of this review include the comprehensive and systematic search in accordance with the PRISMA-P statements and the pre-defined methodology based on the Cochrane Handbook for Systematic Reviews of Interventions. Potential limitations of our review include the quality of the studies due to broad inclusion criteria and possible low number of eligible studies.

\section{Author affiliations}

${ }^{1}$ Anesthesiology and Perioperative Medicine, Queen's University, Kingston, Ontario, Canada

${ }^{2}$ School of Nursing, Faculty of Health Sciences, McMaster University, Hamilton, Ontario, Canada

${ }^{3}$ Division of Cardiology, Medicine, McMaster University Faculty of Health Sciences, Hamilton, Ontario, Canada

${ }^{4}$ Departments of Biomedical and Molecular Sciences, Queen's University Faculty of Health Sciences, Kingston, Ontario, Canada

${ }^{5}$ Departments of Biomedical and Molecular Sciences, Centre for Neuroscience Studies, Queen's University Faculty of Health Sciences, Kingston, Ontario, Canada ${ }^{6}$ School of Policy Studies, Queen's University, Kingston, Ontario, Canada

\section{Twitter Michael McGillion @cardiacpainRC}

Acknowledgements We would like to acknowledge Paola Durando, who is a librarian at Queen's University who assisted in the conducting of the literature search.

Contributors VS wrote the manuscript. IG is the primary investigator, conceived the study concept and was involved in the drafting of the protocol manuscript. JP is a coinvestigator and content expert on heart rate variability. GK, JL, PJD, MM, RA and JP are coinvestigators and content experts in postoperative outcomes. All authors were involved in the editing of the manuscript and have approved the publication of the protocol.

Funding This project was funded, in part, by the CIHR SPOR Chronic Pain Network. Competing interests None declared.

Patient consent for publication Not required.

Provenance and peer review Not commissioned; externally peer reviewed.

Supplemental material This content has been supplied by the author(s). It has not been vetted by BMJ Publishing Group Limited (BMJ) and may not have been peer-reviewed. Any opinions or recommendations discussed are solely those of the author(s) and are not endorsed by BMJ. BMJ disclaims all liability and responsibility arising from any reliance placed on the content. Where the content includes any translated material, BMJ does not warrant the accuracy and reliability of the translations (including but not limited to local regulations, clinical guidelines, terminology, drug names and drug dosages), and is not responsible for any error and/or omissions arising from translation and adaptation or otherwise.

Open access This is an open access article distributed in accordance with the Creative Commons Attribution Non Commercial (CC BY-NC 4.0) license, which permits others to distribute, remix, adapt, build upon this work non-commercially, and license their derivative works on different terms, provided the original work is properly cited, appropriate credit is given, any changes made indicated, and the use is non-commercial. See: http://creativecommons.org/licenses/by-nc/4.0/.
ORCID iD

lan Gilron http://orcid.org/0000-0002-5293-8792

\section{REFERENCES}

1 Weiser TG, Regenbogen SE, Thompson KD, et al. An estimation of the global volume of surgery: a modelling strategy based on available data. Lancet 2008;372:139-44.

2 Sellers D, Srinivas C, Djaiani G. Cardiovascular complications after non-cardiac surgery. Anaesthesia 2018;73:34-42.

3 Laitio T, Jalonen J, Kuusela T, et al. The role of heart rate variability in risk stratification for adverse postoperative cardiac events. Anesth Analg 2007;105:1548-60.

4 Botto F, Alonso-Coello P, Chan MTV, et al. Myocardial injury after noncardiac surgery: a large, international, prospective cohort study establishing diagnostic criteria, characteristics, predictors, and 30day outcomes. Anesthesiology 2014;120:564-78.

5 Haynes AB, Weiser TG, Berry WR, et al. A surgical safety checklist to reduce morbidity and mortality in a global population. $N$ Engl J Med 2009;360:491-9.

6 Kristensen SD, Knuuti J, Saraste A. ESC/ESA guidelines on noncardiac surgery: cardiovascular assessment and management: the joint Task force on non-cardiac surgery: cardiovascular assessment and management of the European Society of Cardiology (ESC) and the European Society of Anaesth. Eur Heart J 2014;2014:2383-431.

7 Devereaux PJ, Sessler DI. Cardiac complications in patients undergoing major noncardiac surgery. $N$ Engl J Med 2015;373:2258-69.

8 Desborough JP. The stress response to trauma and surgery. $\mathrm{Br} \mathrm{J}$ Anaesth 2000;85:109-17.

9 Verbree-Willemsen L, Grobben RB, van Waes JA, et al. Causes and prevention of postoperative myocardial injury. Eur J Prev Cardiol 2019;26:59-67.

10 Abbott TEF, Pearse RM, Archbold RA, et al. A prospective international multicentre cohort study of intraoperative heart rate and systolic blood pressure and myocardial injury after noncardiac surgery: results of the vision study. Anesth Analg 2018;126:1936-45.

11 Devereaux PJ, Szczeklik W. Myocardial injury after non-cardiac surgery: diagnosis and management. Eur Heart J 2019:1-9.

12 Filipovic $M$, Jeger $\mathrm{R}$, Probst $\mathrm{C}$, et al. Heart rate variability and cardiac troponin I are incremental and independent predictors of one-year all-cause mortality after major noncardiac surgery in patients at risk of coronary artery disease. J Am Coll Cardiol 2003;42:1767-76.

13 Martinez EA, Nass CM, Jermyn RM, et al. Intermittent cardiac troponin-I screening is an effective means of surveillance for a perioperative myocardial infarction. J Cardiothorac Vasc Anesth 2005;19:577-82.

14 Duceppe E, Parlow J, MacDonald P, et al. Canadian cardiovascular Society guidelines on perioperative cardiac risk assessment and management for patients who undergo noncardiac surgery. Can $J$ Cardiol 2017;33:17-32.

15 Thygesen K, Alpert JS, Jaffe AS, et al. Third universal definition of myocardial infarction. Eur Heart $J$ 2012;33:2551-67.

16 Puelacher C, Lurati Buse G, Seeberger D, et al. Perioperative myocardial injury after noncardiac surgery: incidence, mortality, and characterization. Circulation 2018;137:1221-32.

17 Devereaux PJ, Duceppe E, Guyatt G, et al. Dabigatran in patients with myocardial injury after non-cardiac surgery (MANAGE): an international, randomised, placebo-controlled trial. Lancet 2018;391:2325-34.

18 Zhang L-J, Li N, Li Y, et al. Cardiac biomarkers predicting MACE in patients undergoing noncardiac surgery: a meta-analysis. Front Physiol 2018;9:1923.

19 Electrophysiology Task Force of the European Society of Cardiology the North American Society of Pacing. Heart rate variability: standards of measurement, physiological interpretation, and clinical use task. Circulation 1996;93:1043-65.

20 Anderson TA. Heart rate variability: implications for perioperative anesthesia care. Curr Opin Anaesthesiol 2017;30:691-7.

21 Heathers JAJ. Everything hertz: methodological issues in short-term frequency-domain HRV. Front Physiol 2014;5:1-15.

22 Nakatsuka I, Ochiai R, Takeda J. Changes in heart rate variability in sevoflurane and nitrous oxide anesthesia: effects of respiration and depth of anesthesia. J Clin Anesth 2002;14:196-200.

23 Galletly DC, Buckley DH, Robinson BJ, et al. Heart rate variability during propofol anaesthesia. Br J Anaesth 1994;72:219-20.

24 Parlow JL, van Vlymen JM, Odell MJ. The duration of impairment of autonomic control after anticholinergic drug administration in humans. Anesth Analg 1997;84:155-9. 
25 Parlow JL, Bégou G, Sagnard P, et al. Cardiac baroreflex during the postoperative period in patients with hypertension: effect of clonidine. Anesthesiology 1999;90:681-92.

26 Nault MA, Milne B, Parlow JL. Effects of the selective $\mathrm{H} 1$ and $\mathrm{H} 2$ histamine receptor antagonists loratadine and ranitidine on autonomic control of the heart. Anesthesiology 2002;96:336-41.

27 Chenier-Hogan N, Brown CA, Hains SMJ, et al. Heart rate variability response to standing in men and women receiving $\mathrm{d}$,l-sotalol following coronary artery bypass graft surgery. Biol Res Nurs 2012;14:38-47.

28 Filipovic M, Jeger RV, Girard T, et al. Predictors of long-term mortality and cardiac events in patients with known or suspected coronary artery disease who survive major non-cardiac surgery. Anaesthesia 2005;60:5-11.

29 Buccelletti E, Gilardi E, Scaini E, et al. Heart rate variability and myocardial infarction: systematic literature review and metanalysis. Eur Rev Med Pharmacol Sci 2009;13:299-307.

30 Cortelli P, Pierangeli G. Chronic pain-autonomic interactions. Neurol Sci 2003;24:s68-70.

31 Schlereth T, Birklein F. The sympathetic nervous system and pain Neuromolecular Med 2008;10:141-7.

32 Koenig J, Falvay D, Clamor A, et al. Pneumogastric (vagus) nerve activity indexed by heart rate variability in chronic pain patients compared to healthy controls: a systematic review and metaanalysis. Pain Physician 2016;19:E55-78.

33 Masel EK, Huber P, Engler T, et al. Heart rate variability during treatment of breakthrough pain in patients with advanced cancer: a pilot study. J Pain Res 2016;9:1215-20.

34 Terkelsen AJ, Mølgaard H, Hansen J, et al. Heart rate variability in complex regional pain syndrome during rest and mental and orthostatic stress. Anesthesiology 2012;116:133-46.

35 Mork PJ, Nilsson J, Lorås HW, et al. Heart rate variability in fibromyalgia patients and healthy controls during non-REM and REM sleep: a case-control study. Scand J Rheumatol 2013;42:505-8.

36 Kang J-H, Chen $\mathrm{H}-\mathrm{S}$, Chen S-C, et al. Disability in patients with chronic neck pain. Clin J Pain 2012;28:797-803.

37 Koenig J, Jarczok MN, Ellis RJ, et al. Heart rate variability and experimentally induced pain in healthy adults: a systematic review. Eur J Pain 2014;18:301-14.

38 Boselli E, Daniela-lonescu M, Bégou G, et al. Prospective observational study of the non-invasive assessment of immediate postoperative pain using the analgesia/nociception index (ANI). $\mathrm{Br} J$ Anaesth 2013;111:453-9.
39 Gall O, Champigneulle B, Schweitzer B, et al. Postoperative pain assessment in children: a pilot study of the usefulness of the analgesia nociception index. Br J Anaesth 2015;115:890-5.

40 Koenig J, Jarczok MN, Ellis RJ, et al. Lowered parasympathetic activity in apparently healthy subjects with self-reported symptoms of pain: preliminary results from a pilot study. Pain Pract 2015;15:314-8.

41 Treister R, Kliger M, Zuckerman G, et al. Differentiating between heat pain intensities: the combined effect of multiple autonomic parameters. Pain 2012;153:1807-14.

42 Appelhans BM, Luecken LJ. Heart rate variability and pain: associations of two interrelated homeostatic processes. Biol Psychol 2008;77:174-82.

43 Fujiwara Y, Kurokawa S, Shibata Y, et al. Sympathovagal effects of spinal anaesthesia with intrathecal or intravenous fentanyl assessed by heart rate variability. Acta Anaesthesiol Scand 2009;53:476-82.

44 Storella RJ, Kandell RB, Horrow JC, et al. Nonlinear measures of heart rate variability after fentanyl-based induction of anesthesia. Anesth Analg 1995;81:1292-4.

45 Galletly DC, Westenberg AM, Robinson BJ, et al. Effect of halothane, isoflurane and fentanyl on spectral components of heart rate variability. Br J Anaesth 1994;72:177-80

46 Lafreniere G, Milne B, Brunet DG, et al. Autonomic circulatory and cerebrocortical responses during increasing depth of propofol sedation/hypnosis in humans. Can J Anaesth 2000;47:441-8.

47 Kato M, Komatsu T, Kimura T, et al. Spectral analysis of heart rate variability during isoflurane anesthesia. Anesthesiology 1992;77:669-74

48 Headrick JP, Pepe S, Peart JN. Non-analgesic effects of opioids: cardiovascular effects of opioids and their receptor systems. Curr Pharm Des 2012;18:6090-100.

49 Cashman JN, Dolin SJ. Respiratory and haemodynamic effects of acute postoperative pain management: evidence from published data. Br J Anaesth 2004:93:212-23.

50 Moher D, Liberati A, Tetzlaff J, et al. Preferred reporting items for systematic reviews and meta-analyses: the PRISMA statement. PLoS Med 2009;6:e1000097.

51 Sterne JA, Gavaghan D, Egger M. Publication and related bias in meta-analysis: power of statistical tests and prevalence in the literature. J Clin Epidemiol 2000;53:1119-29.

52 Sterne J, Hernán M, McAleenan A. Assessing risk of bias in a non-randomized study (Chapter 25). In: Cochrane handbook for systematic reviews of interventions version 6.0, 2019. 